\title{
A criterion to justify a holomorphic function
}

\author{
Feng $\mathbf{Q i}^{1,2,3,{ }^{*}}$ and Bai-Ni Guo ${ }^{4}$ \\ ${ }^{1}$ Institute of Mathematics, Henan Polytechnic University, Jiaozuo City, Henan Province, 454010, China \\ ${ }^{2}$ College of Mathematics, Inner Mongolia University for Nationalities, Tongliao City, Inner Mongolia Autonomous Region, 028043, China \\ ${ }^{3}$ Department of Mathematics, College of Science, Tianjin Polytechnic University, Tianjin City, 300387, China \\ ${ }^{4}$ School of Mathematics and Informatics, Henan Polytechnic University, Jiaozuo City, Henan Province, 454010, China \\ *Corresponding author E-mail: qifeng618@gmail.com
}

\begin{abstract}
In the paper, the authors present a criterion, free of considering differentiability and any partial derivative, to justify a holomorphic function and illustrate the criterion by several examples.
\end{abstract}

Keywords: Criterion; Justifying; Holomorphic Function; Differentiability; Partial Derivative; Example.

\section{Main result}

In the theory of complex functions, the usually used criterion to justify a holomorphic function is considering differentiability of real and imaginary parts and solvability of system of the CauchyRiemann equations for a complex function.

The main aim of this paper is to present a criterion which can be summarized as Theorems 1.1 and 1.2 below, free of considering differentiability and any partial derivative, to justify a holomorphic function.

Theorem 1.1. Let $D \subseteq \mathbb{C}$ be a domain such that the origin $0 \in D$. Then a complex function

$f(z)=f(x+i y)=u(x, y)+i v(x, y)$

is holomorphic on $D$ if and only if the functions

$u(z, 0), \quad v(z, 0), \quad u(0,-i z), \quad$ and $\quad v(0,-i z)$

are holomorphic and system of equations

$\left\{\begin{array}{l}f(z)=u(z, 0)+i v(z, 0) \\ f(z)=u(0,-i z)+i v(0,-i z)\end{array}\right.$

is valid on the domain $D$.

Theorem 1.2. Let $D \subseteq \mathbb{C}$ be a domain and $z_{0}=x_{0}+i y_{0} \in D$. Then a complex function $f(z)$ expressed in (1.1) is holomorphic on $D$ if and only if the functions

$$
\begin{array}{cc}
u\left(z-i y_{0}, y_{0}\right), & v\left(z-i y_{0}, y_{0}\right), \\
u\left(x_{0}, i\left(x_{0}-z\right)\right), & v\left(x_{0}, i\left(x_{0}-z\right)\right)
\end{array}
$$

are holomorphic and the system of equations

$$
\left\{\begin{array}{l}
f(z)=u\left(z-i y_{0}, y_{0}\right)+i v\left(z-i y_{0}, y_{0}\right) \\
f(z)=u\left(x_{0}, i\left(x_{0}-z\right)\right)+i v\left(x_{0}, i\left(x_{0}-z\right)\right)
\end{array}\right.
$$

is valid on the domain $D$.
In next section, we will prove Theorems 1.1 and 1.2.

In the final section, we will take several examples, which are much familiar for readers, to illustrate Theorems 1.1 and 1.2.

Theorems 1.1 and 1.2 generalize [3, pp. 182-183, Exercise 7].

\section{Proofs of Theorems $\mathbf{1 . 1}$ and $\mathbf{1 . 2}$}

Proof of Theorem 1.1. Necessity. Taylor's theorem [1, p. 177] reads that, if $f(z)$ is holomorphic in the region $\Omega$, containing $z_{0}$, then the representation

$f(z)=\sum_{k=0}^{\infty} \frac{f^{(k)}\left(z_{0}\right)}{k !}\left(z-z_{0}\right)^{k}$

is valid in the largest open disk of center $z_{0}$ contained in $\Omega$. Letting $z_{0}=0$ and assuming $\frac{f^{(k)}(0)}{k !}=A_{k}+i B_{k}$ lead to

$u(x, y)+i v(x, y)=\sum_{k=0}^{\infty}\left(A_{k}+i B_{k}\right)(x+i y)^{k}$.

Taking $y=0$ and $x=0$ respectively in the above equation gives

$u(x, 0)+i v(x, 0)=\sum_{k=0}^{\infty}\left(A_{k}+i B_{k}\right) x^{k}$

and

$u(0, y)+i v(0, y)=\sum_{k=0}^{\infty}\left(A_{k}+i B_{k}\right)(i y)^{k}$.

Consequently, it follows that

$u(x, 0)=\sum_{k=0}^{\infty} A_{k} x^{k}, \quad v(x, 0)=\sum_{k=0}^{\infty} B_{k} x^{k}$,

$u(0, y)=\sum_{k=0}^{\infty}(-1)^{k} A_{2 k} y^{2 k}+\sum_{k=0}^{\infty}(-1)^{k+1} B_{2 k+1} y^{2 k+1}$,

$v(0, y)=\sum_{k=0}^{\infty}(-1)^{k+1} A_{2 k+1} y^{2 k+1}+\sum_{k=0}^{\infty}(-1)^{k} B_{2 k} y^{2 k}$. 
This implies, by Taylor's theorem and the important uniqueness theorem (see [1, p. 279, Theorem 1]) that the four functions in (1.2) are holomorphic.

Letting $y=0$ and $x=0$ respectively in (1.1) reveals that the equation system

$$
\left\{\begin{array}{l}
f(x)=u(x, 0)+i v(x, 0) \\
f(i y)=u(0, y)+i v(0, y)
\end{array}\right.
$$

is valid for $x, y \in(-\delta, \delta)$, where $\delta>0$. By the important uniqueness theorem and by holomorphic property of the four functions in (1.2), we see that the system (2.2) is valid on the whole domain $D$. Consequently, replacing $x$ by $z$ and $y$ by $-i z$ in (2.2) figures out the equation system (1.3).

The sufficiency is obvious. Theorem 1.1 is complete.

Proof of Theorem 1.2. If the origin 0 does not contain in the domain $D$ and the complex function $f(z)$ is holomorphic on $D$, then we can consider the function

$$
\begin{aligned}
& F(w)=f\left(w+z_{0}\right)=f\left(p+x_{0}+i\left(q+y_{0}\right)\right) \\
& =u\left(p+x_{0}, q+y_{0}\right)+i v\left(p+x_{0}, q+y_{0}\right)=u_{z_{0}}(p, q)+i v_{z_{0}}(p, q)
\end{aligned}
$$

for

$w=p+i q \in D_{z_{0}}=\left\{z-z_{0}: z_{0}, z \in D\right\}$.

When we apply Theorem 1.1 to both the function $F(w)$ and the domain $D_{z_{0}}$, the equation system (1.3) becomes

$$
\left\{\begin{array}{l}
F(w)=u_{z_{0}}(w, 0)+i v_{z_{0}}(w, 0) \\
F(w)=u_{z_{0}}(0,-i w)+i v_{z_{0}}(0,-i w)
\end{array}\right.
$$

which is equivalent to

$$
\left\{\begin{array}{l}
f\left(w+z_{0}\right)=u\left(w+x_{0}, y_{0}\right)+i v\left(w+x_{0}, y_{0}\right) \\
f\left(w+z_{0}\right)=u\left(x_{0}, y_{0}-i w\right)+i v\left(x_{0}, y_{0}-i w\right)
\end{array}\right.
$$

for $w=p+i q \in D_{z_{0}}$. Replacing $w$ by $z-z_{0}$ in the last equation system results in

$$
\left\{\begin{array}{l}
f(z)=u\left(z-z_{0}+x_{0}, y_{0}\right)+i v\left(z-z_{0}+x_{0}, y_{0}\right) \\
f(z)=u\left(x_{0}, y_{0}-i\left(z-z_{0}\right)\right)+i v\left(x_{0}, y_{0}-i\left(z-z_{0}\right)\right)
\end{array}\right.
$$

for $z \in D$. Simplifying the above equations lead to (1.5). The proof of Theorem 1.2 is complete.

\section{Examples}

To illustrate the criterion, we list several examples below.

\subsection{Example}

It is common knowledge in the theory of complex functions that the complex functions

$$
\bar{z}=x-i y, \quad|z|=\sqrt{x^{2}+y^{2}}, \quad \mathfrak{R}(z)=x, \quad \text { and } \quad \mathfrak{I}(z)=y
$$

are not holomorphic everywhere on the whole complex plane $\mathbb{C}$. This can be verified by respectively considering solvability of the CauchyRiemann equations and differentiability of real and imaginary parts of the four functions in (3.1).

With the help of Theorem 1.1, due to the obvious fact that the equation systems

$$
\left\{\begin{array} { l } 
{ \overline { z } = z ; } \\
{ \overline { z } = - z , }
\end{array} \quad \left\{\begin{array} { l } 
{ | z | = \sqrt { z ^ { 2 } } ; } \\
{ | z | = \sqrt { - z ^ { 2 } } , }
\end{array} \quad \left\{\begin{array}{l}
\Re(z)=z ; \\
\Re(z)=0
\end{array}\right.\right.\right.
$$

and

$\left\{\begin{array}{l}\mathfrak{I}(z)=0 \\ \mathfrak{I}(z)=-i z\end{array}\right.$

are respectively valid only at the origin 0 , we immediately see that all of the four functions in (3.1) are not holomorphic everywhere on the complex plane $\mathbb{C}$.

The above arguments show that Theorem 1.1 provides a simpler criterion, without computing derivatives, to justify holomorphic functions.

\subsection{Example}

Let

$f(z)=u(x, y)+i v(x, y)=x^{2}+a x y+b y^{2}+i\left(\alpha x^{2}+\beta x y+y^{2}\right)$

on $\mathbb{C}$. By virtue of the Cauchy-Riemann equations, we can obtain that, when

$a=2, \quad b=-1, \quad \alpha=-1, \quad$ and $\quad \beta=2$,

the function $f(z)$ is holomorphic on $\mathbb{C}$. See [2, p. 43, Example 2]. Since

$u(z, 0)=z^{2}, \quad v(z, 0)=\alpha z^{2}, \quad u(0,-i z)=-b z^{2}$,

and

$v(0,-i z)=-z^{2}$,

the equation system (1.3) becomes

$\left\{\begin{array}{l}x^{2}+a x y+b y^{2}+i\left(\alpha x^{2}+\beta x y+y^{2}\right)=(1+\alpha i) z^{2} ; \\ x^{2}+a x y+b y^{2}+i\left(\alpha x^{2}+\beta x y+y^{2}\right)=-(b+i) z^{2}\end{array}\right.$

which is a linear system of equations in four variables $a, b, \alpha$, and $\beta$. The equation system (3.4) has the unique solution (3.3). By Theorem 1.1, we reveal that, under the condition (3.3), the function $f(z)$ in (3.2) is holomorphic and can be easily rewritten in terms of $z$, rather than $x$ and $y$, as

$f(z)=(1-i) z^{2}$

on the whole complex plane $\mathbb{C}$.

The arguments tell us that, to justify a holomorphic function $f(z)$, it is sufficient to solve the equation system (1.3).

\subsection{Example}

It is general knowledge that the logarithmic function

$f(z)=\ln z=\ln |z|+i \arg z$

is holomorphic on the cut plane $\mathscr{A}=\mathbb{C} \backslash(-\infty, 0]$.

Taking any point $z_{0}=x_{0}+y_{0} \in \mathscr{A}$ and replacing in (1.5) $f(z)$ by the logarithmic function $\ln z$ gives

$\left\{\begin{array}{l}\ln z=\ln \left|z-i y_{0}+i y_{0}\right|+i \arg \left(z-i y_{0}+i y_{0}\right)=\ln z \\ \ln z=\ln \left|x_{0}+i \times i\left(x_{0}-z\right)\right|+i \arg \left(x_{0}+i \times i\left(x_{0}-z\right)\right)=\ln z\end{array}\right.$

which is valid on the cut plane $\mathscr{A}$. This implies, by Theorem 1.2, that the logarithmic function $\ln z$ is holomorphic on the cut plane $\mathscr{A}$. Since the imaginary part of $\ln z$ is $v(x, y)=\arg z=\arg (x+i y)$ on $\mathscr{A}$, by Theorem 1.2, we further obtain that, for any $z_{0}=x_{0}+i y_{0} \in \mathscr{A}$, the functions

$v\left(z-i y_{0}, y_{0}\right)=\arg \left(z-i y_{0}+i y_{0}\right)=\arg z$

and

$v\left(x_{0}, i\left(x_{0}-z\right)\right)=\arg \left(x_{0}-\left(x_{0}-z\right)\right)=\arg z$

are holomorphic on $\mathscr{A}$. Simply speaking, the $\operatorname{argument} \arg z$ is holomorphic on $\mathscr{A}$. 


\subsection{Example}

Let

$f(z)=\sqrt{1+z}+\sqrt{1-z}$

on

$D=\mathbb{C} \backslash(-\infty,-1] \cup[1, \infty)$.

It is a single-valued complex function clearly. See [1, p. 73, Exercise 1].

A direct computation gives

$$
\begin{aligned}
& f(x+i y)= u(x, y)+i v(x, y) \\
&= \sqrt[4]{(x-1)^{2}+y^{2}} \cos \frac{\arg (1-x-i y)}{2} \\
&+\sqrt[4]{(x+1)^{2}+y^{2}} \cos \frac{\arg (1+x+i y)}{2} \\
&+i\left[\sqrt[4]{(x-1)^{2}+y^{2}} \sin \frac{\arg (1-x-i y)}{2}\right. \\
&\left.+\sqrt[4]{(x+1)^{2}+y^{2}} \sin \frac{\arg (1+x+i y)}{2}\right] \\
& u(z, 0)=\sqrt[4]{(z-1)^{2}} \cos \frac{\arg (1-z)}{2}+\sqrt[4]{(z+1)^{2}} \cos \frac{\arg (z+1)}{2}, \\
& v(z, 0)=\sqrt[4]{(z-1)^{2}} \sin \frac{\arg (1-z)}{2}+\sqrt[4]{(z+1)^{2}} \sin \frac{\arg (z+1)}{2}, \\
& u(0,-i z)= \sqrt[4]{1-z^{2}}\left[\cos \frac{\arg (1-z)}{2}+\cos \frac{\arg (1+z)}{2}\right] \\
& v(0,-i z)= \sqrt[4]{1-z^{2}}\left[\sin \frac{\arg (1-z)}{2}+\sin \frac{\arg (1+z)}{2}\right]
\end{aligned}
$$

and the validity of the equation system (1.3) applying to the function (3.5) on $D$. Consequently, by virtue of Theorem 1.1, we see that the function (3.5) is holomorphic on the domain (3.6).

\subsection{Example}

Suppose $f(z)=u(x, y)+i v(x, y)$ and its conjugate $\overline{f(z)}=u(x, y)-$ $i v(x, y)$ are all holomorphic on a domain $D$ and let $z_{0}=x_{0}+i y_{0} \in D$. By virtue of Theorem 1.2, we see that the functions in (1.4) are holomorphic and that the equation systems (1.5) and

$$
\left\{\begin{array}{l}
\overline{f(z)}=u\left(z-i y_{0}, y_{0}\right)-i v\left(z-i y_{0}, y_{0}\right) ; \\
\overline{f(z)}=u\left(x_{0}, i\left(x_{0}-z\right)\right)-i v\left(x_{0}, i\left(x_{0}-z\right)\right)
\end{array}\right.
$$

are valid. Hence, it follows that

$$
\begin{aligned}
u(x, y)-i v(x, y) & =\overline{u\left(z-i y_{0}, y_{0}\right)}-\overline{i v\left(z-i y_{0}, y_{0}\right)} \\
& =u\left(z-i y_{0}, y_{0}\right)-i v\left(z-i y_{0}, y_{0}\right) \\
& =\overline{u\left(x_{0}, i\left(x_{0}-z\right)\right)}-i \overline{v\left(x_{0}, i\left(x_{0}-z\right)\right)} \\
& =u\left(x_{0}, i\left(x_{0}-z\right)\right)-i v\left(x_{0}, i\left(x_{0}-z\right)\right) .
\end{aligned}
$$

As a result, the functions in (1.4) are real constants on $D$, and so the complex function $f(z)$ and its conjugate $\overline{f(z)}$ are two constants.

\section{References}

[1] L. V. Ahlfors, Complex analysis: An introduction of the theory of analytic functions of one complex variable, 2nd ed., McGraw-Hill Book Co., New York-Toronto-London, 1966.

[2] Teaching and Research Section of Higher Mathematics at Xi' an Jiaotong University, Complex Functions, 4th ed., Higher Education Press, Beijing, China, 1996. (Chinese)

[3] Y.-Q. Zhong, Theory of Complex Functions, 3rd. ed., Higher Education Press, Beijing, China, 2004. (Chinese) 